\title{
An in situ TEM Study of Ferroelastic Domain Mobility
}

Peng Gao ${ }^{1}$, Jacob R. Jokisaari ${ }^{1}$, Christopher T. Nelson ${ }^{1}$, Seung-Hyub Baek ${ }^{2}$, Morgan Trassin ${ }^{3}$, Chung Wung Bark ${ }^{2}$, Ramamoorthy Ramesh ${ }^{3}$, Chang-Beom Eom ${ }^{2}$, and Xiaoqing Pan ${ }^{1}$

1. Department of Materials Science and Engineering, University of Michigan, Ann Arbor, Michigan 48109, USA

2. Department of Materials Science and Engineering, University of Wisconsin-Madison, Madison, Wisconsin 53706, USA

3. Department of Materials Science and Engineering, University of California, Berkeley, Berkeley, CA 94720, USA

Ferroelectric materials, such as lead zirconate titanate (PZT), have a wide range of applications in devices due to their large electromechanical response. Ferroelastic domain switching in ferroelectrics, which alters polarization and strain states simultaneously, could significantly enhance the electromechanical response. For tetragonal perovskite ferroelectrics, first-principles calculations suggest that the barrier to motion is much lower for ferroelastic domain ( $a$-domain) walls than for $180^{\circ}$ domain walls. However, many experimental studies show ferroelastic domain walls in PZT to be immobile in thin films. This discrepancy is likely due to interface clamping and/or defect pinning in real samples.

We use in situ transmission electron microscopy (TEM) to study the structure and dynamic response of domains to applied electric field in a cross-section sample of a tetragonal $\mathrm{Pb}\left(\mathrm{Zr}_{0.2} \mathrm{Ti}_{0.8}\right) \mathrm{O}_{3}$ thin film. The PZT thin films of $200 \mathrm{~nm}$ in thickness were grown on (110)- $\mathrm{DyScO}_{3}$ substrates with a $\mathrm{SrRuO}_{3}$ (SRO) buffer layer, which acts as a conductive electrode. As-grown films were predominantly c-axis oriented, but with many $a$-domains to accommodate the lattice parameter mismatch between the thin film and substrate. The $a$-domains occurred in three types: those that extend from interface to surface, those that extend from the surface to terminate in the middle of the film, and those that extend from the bottom interface and terminate in the film. We found that the response of $a$-domains to an external electric field depends on their terminating positions within the film. Those $a$-domains terminating in the middle of the film are always active in electric fields as they adopt a classic needle-like shape to minimize the elastic/electrostatic energy at their forward edge [1], where the barrier to polarization rotation is lowest because they cannot maintain a head-tail arrangement of the polarization vectors. Fig.1a shows that partial $a$-domains originating at the free surface can be erased permanently with a moderate electric field, while Fig. $1 \mathrm{~b}$ indicates those $a$-domains extending from the bottom interface can be switched only partially and return to their previous state after the field is removed. Any $a$-domains extending through the thickness of the film are less mobile due to interface clamping and the absence of the high energy domain front. In this case, the threshold for domain wall motion usually exceeds the breakdown field of the film, as shown in Fig. 1c. Furthermore, the $a$-domains located at bottom interface are usually pinned by dislocations $[2,3]$ as shown in Fig.2. These misfit dislocations may also account for immobility of some $a$-domains as the strain field near a dislocation core favors the in-plane polarization.

In conclusion, these studies suggest that the mobility of ferroelastic domains in PZT thin films depends strongly on the local structure and defects. These results provide an important mechanism to understand the controversy regarding the mobility of ferroelectric and ferroelastic domains. 
References:

[1] Little, E. A. Physical Review 98 (1955) 978.

[2] Kiguchi, T. Science and Technology of Advanced Materials 12 (2011) 034413.

[2] Su, D. Appl. Phys. Lett. 99 (2011) 102902.

[4] The authors gratefully acknowledge the financial support through DOE grant DoE/BES DE-FG0207ER46416 and NSF DMR-0907191.

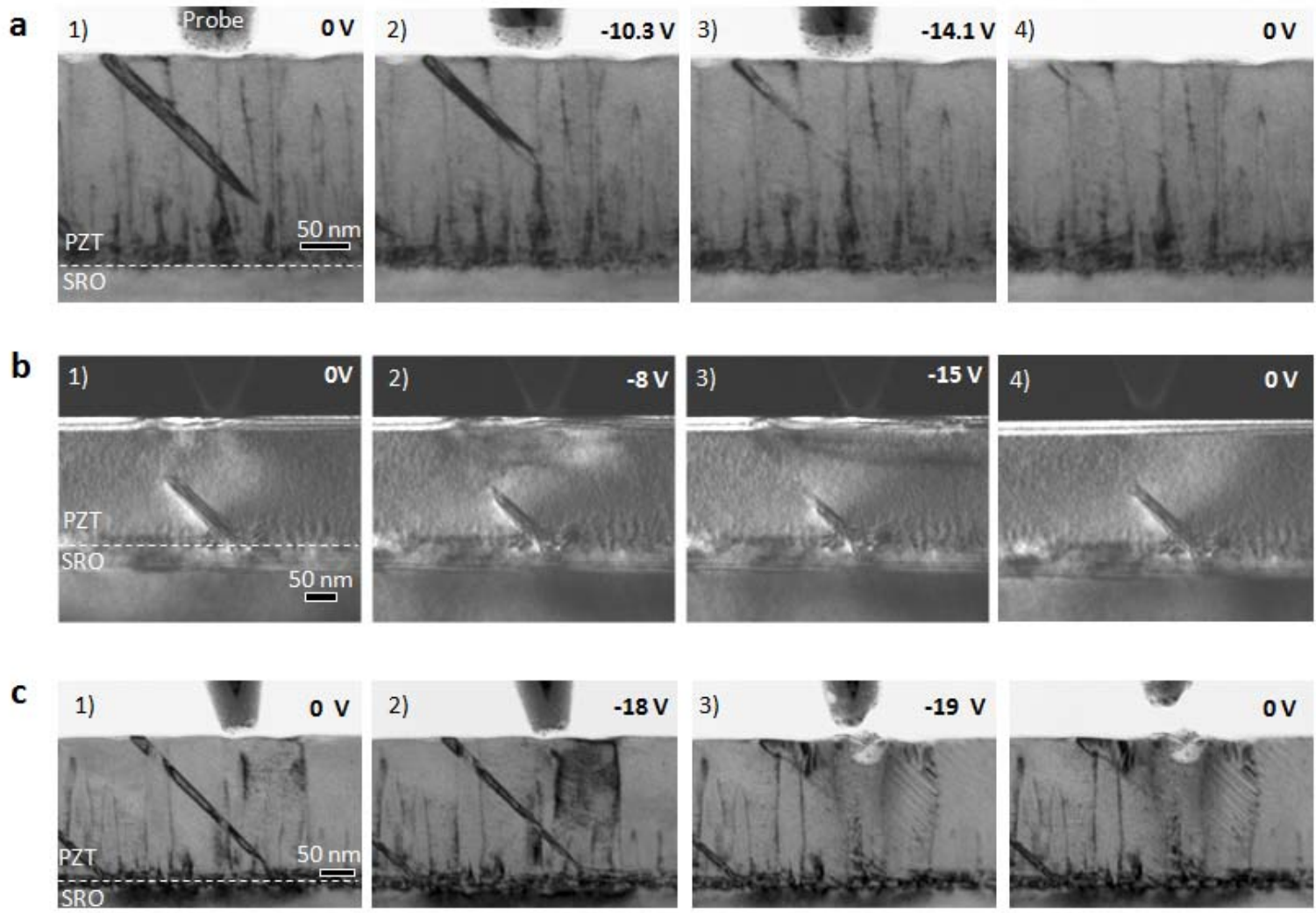

Figure 1. Dynamic evolution of ferroelastic domains in a PZT thin film under applied electrical fields. (a) A partial $a$-domain sitting at free surface. (b) A partial $a$-domain sitting at the bottom interface. (c) A full $a$-domain extending through the thickness of the film.
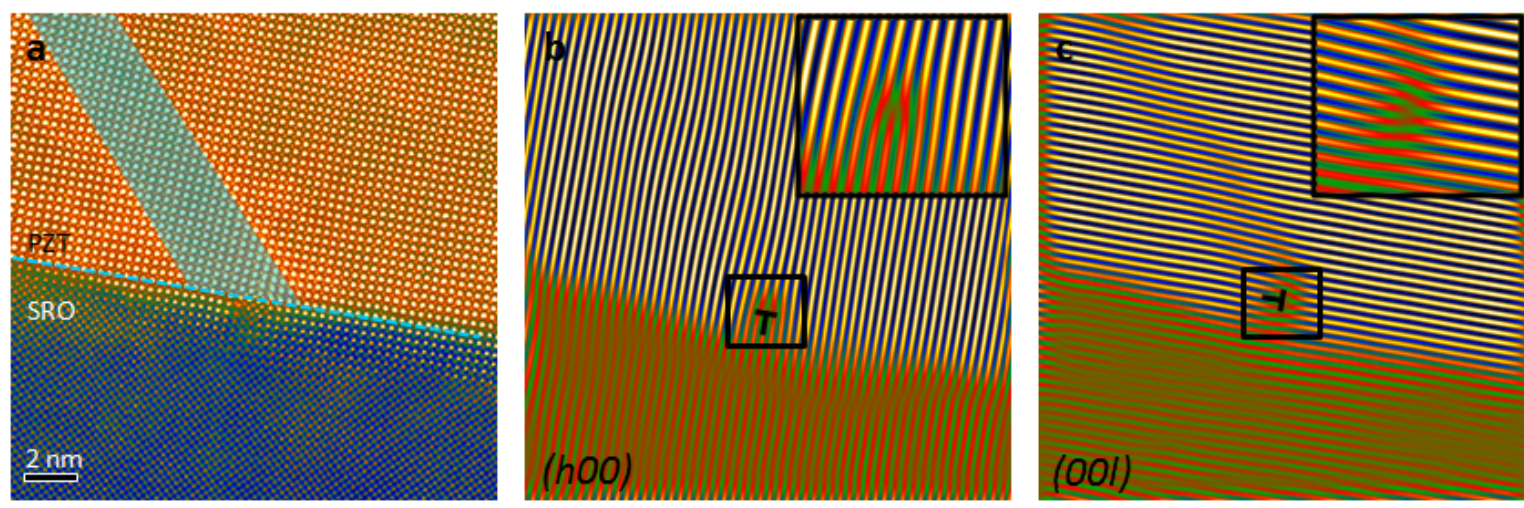

Figure 2. An $a$-domain pinned by misfit dislocations at the bottom interface. (a) High resolution high angle angular dark field image of an $a$-domain at the bottom interface between PZT and SRO. The same image filtered in Fourier space by including only the (h00) (b) and the (00l) (c) lattice frequencies. The labeled dislocation cores are magnified in the up-right corners. 